Funding: GlaxoSmithKline, Waltham, MA, USA

Encore statement: This data is presented on behalf of the original authors with their permission. Presented at European Society for Medical Oncology (ESMO) annual meeting, September 19-21, 2020, Virtual.

Dr. Kristeleit reports personal fees from Tesaro.

Dr. Mathews reports institutional grants from Tesaro.

Dr. Redondo reports institutional research funding from PharmaMar, Roche, and Eisai; and advisory roles at PharmaMar, AstraZeneca, Tesaro, Roche, and Eisai.

Dr. Brown reports honoraria from Olympus; consulting or advisory role at Caris, Tesaro, Clovis, AstraZeneca, and Genentech; and speakers' bureau at Clovis.

Drs. Huang, Eliason, and $\mathrm{Im}$ are employees of GlaxoSmithKline.

\section{CANCER CELL STEMNESS AND COLLAGEN REGULATORS OF STEMNESS IN UTERINE SEROUS CARCINOMA (USC) MIRROR OVARIAN SEROUS CANCER (OSC) CELL STEMNESS: EVIDENCE EMERGING FROM ARK1-USC}

${ }^{1}$ Hartmut Hanauske-Abel, ${ }^{2}$ Sukwinder Singh, ${ }^{3}$ Mainul Hoque, ${ }^{3}$ Seema Husain, ${ }^{4}$ AxelRainer Hanauske, ${ }^{3}$ Patricia Soteropoulos, ${ }^{5}$ Bernadette M Cracchiolo. ${ }^{1}$ Rutgers Njms; Ob/ Gyn/Reprod Health, Microbiol/Biochem/Mol Genet, Peds; ${ }^{2}$ Rutgers Njms; Path/Lab Med; ${ }^{3}$ Rutgers Njms; Microbiol/Biochem/Med Genet; ${ }^{4}$ Technische Universität; Medicine 3; ${ }^{5}$ Rutgers Njms; Ob/Gyn/Reprod Health

\subsection{6/ijgc-2020-ESG0.73}

Introduction/Background ARK1-USC, a highly annotated USCderived cell line with a clinically relevant mutation spectrum, ${ }^{1}$ is employed in vitro and in vivo for translational studies of novel USC therapies. ${ }^{2}$ In ovarian cancer, stemness and malignancy-supporting collagen microenvironment coincide. Both promote resistance to therapy. Considering the shared histological and molecular characteristics of USC and OSC, we hypothesized that USC cells likewise display ovarian markers for stemness and collagen regulators of stemness. We tested this prediction in ARK1-USC.

Methodology Profiling of the time-dependent transcriptome, with flow cytometric analysis of select protein markers.

Results ARK1-USC expressed the repertoire of cancer stem cell (CSC) markers of ovarian malignancies, such as CD44, CD117, CD144, CD133, ROR1, and ALDH1A1. Relative to $12 \mathrm{~h}$ after plating, at $48 \mathrm{~h}$ expression was decreased (CD117 and ROR1 by half, FDR adjusted p-value [q] $\leq 0.003)$; increased (ALDH1A1 and CD144 2.5- and 2.1-fold ( $q=0.027$ and $\mathrm{q} \leq 0.000$, resp.); or unchanged (CD44 and CD133). ARK1-USC also expressed i) the stemness-maintaining COL18, shown in $\mathrm{OSC}^{3}$; and ii) the malignant microenvironment collagen types VI and XI, COL6A1/COL6A2/COL6A3 and COL11A1/COL11A2, shown in OSC to cause platinum resistance $^{4}$ and poor prognosis. ${ }^{5}$ Using fluorophore-labelled monospecific antibodies, flow cytometry confirmed collagen type VI and XVIII production by ALDH1A1 expressing ARK1-USC. In contradistinction to ovarian cancer, ARK1-USC expressed the CSC marker nerve growth factor receptor (NGFR), increasing 3 -fold by $48 \mathrm{~h}(\mathrm{q}=0.039)$; nerve growth factor (NGF) was not expressed at any time. Nevertheless, multiple genes regulating neurogenesis, synaptic plasticity and excitation, axonal transport, and neuron epigenetic reprogramming were expressed, in addition to an inventory of neuronal receptors for neurotransmitters like acetylcholine (e.g. CHRNA3/
CHRNA4/CHRNA5), dopamine/epinephrine/norepinephrine (e. g. ADRA1B/ADRA2C/ADRB1/ADRB2/DDR2/DRD4), serotonin (e.g. HTR2B, HTR6) and the enzymes required for their synthesis (e.g. TPH2, TH).

Conclusion ARK1-USC classify as CSCs with neuronoid propensity. We propose that in reaction to therapies in vivo, a cancer cell subpopulation stabilized in its proper niche of malignant matrix can temporarily differentiate into neuron-like non-proliferative cells endowed with enhanced chemo- and radiation-resistance. This conceptual framework, which captures the current clinical experience with USC treatment, is worthy of further study as it envisions a previously unnoted cytological sanctuary that still holds promising novel mechanistic targets for interdicting cancer cell entry and persistence. Disclosures The authors have nothing to disclose.

\section{REFERENCES}

1. Zhao, et al. (2013) PNAS 110: 2916-2921.

2. Bonazzoli, et al. (2018) Clin Cancer Res 24: 4845-4853.

3. Guenther, et al. (2001) Brit J Cancer 85: 1540-1545.

4. Park, et al. (2013) EMBO Mol Med 5:935-948.

5. Wu, et al. (2014) Oncogene 3: 3432-3440.

\section{DETECTION OF THE SENTINEL LYMPH NODE BY ECOGUIDED MYOMETRIAL INJECTION (TUMIR) OF RADIOTRACER VERSUS HYBRID TRACER (RADIOTRACER- ICG) IN PATIENTS WITH INTERMEDIATE/HIGH RISK ENDOMETRIAL CANCER}

${ }^{1}$ Núria Agustí, ${ }^{1}$ Sergi Vidal-Sicart, ${ }^{2}$ Ariel Gustavo Glickman, ${ }^{2}$ Berta Diaz-Feijoo, ${ }^{2}$ Pere Fusté, ${ }^{2}$ Jaume Pahisa, ${ }^{1}$ Núria Carreras, ${ }^{3}$ Marta Del Pino, ${ }^{2}$ Aureli Torne, ${ }^{1}$ Pilar Paredes. ${ }^{1}$ Hospital Clinic Barcelona; ${ }^{2}$ Hospital Clínic Barcelona; Gynaecological Oncology Unit; ${ }^{3}$ Hospital Clínic; Hospital Clínic Barcelona; Gynaecology

\subsection{6/ijgc-2020-ESG0.74}

Introduction/Background Sentinel lymph node (SLN) detection in patients with endometrial cancer (EC) is usually performed with a $[99 \mathrm{mTc}] \mathrm{Tc}$-albumin nanocolloid radiotracer (RTs). The transvaginal ultrasound-guided myometrial injection of radiotracer, unlike cervical injection, is more representative of tumor's drainage and obtains a higher percentage of SLN. Recently, the use of Indocyanine green (ICG) has gained relevance, although with this technique no pre-surgical lymphatic map is available. The hybrid tracer with RT-ICG could be an alternative to conserve the advantages of both components. The objective of this study is to see the performance of the detection of SLN with RT vs RT-CGI using the TUMIR technique in patients with $\mathrm{EC}$ at risk.

Methodology It is a retrospective study which has included patients with stage I/II CE, high/intermediate risk. Detection of SLN has been performed using the TUMIR technique (figure 1) with $\mathrm{RT}(8 \mathrm{ml}$ with $6 \mathrm{mCi}$ of $\mathrm{RT}$ ) between 2006 and 2017 or hybrid tracer RT-ICG $(4 \mathrm{ml}$ with $6 \mathrm{mCi}$ of RT 0.05 $\mathrm{ml}$ of ICG $(25 \mathrm{mg} / 5 \mathrm{ml})$ ) between 2014 and 2019. A planar and tomographic lymphoscintigraphy (SPECT/CT) has been performed preoperatively (figure 2). After detection and excision of the SLN, a systematic pelvic and paraortic lymphadenectomy has been performed. The histological study of the SLN has been performed by H\&E and IHC.

Results A total of 155 patients have been included (102 with RT and 53 with ICG-RT). The intraoperative SLN detection in the RT group was $79.4 \% \quad(92.6 \%$ of pelvic drainage, $45.7 \%$ of paraortic drainage and $7.4 \%$ exclusively paraortic). A bilateral drainage was found in $32 \%$ of the cases. A $19.6 \%$ 


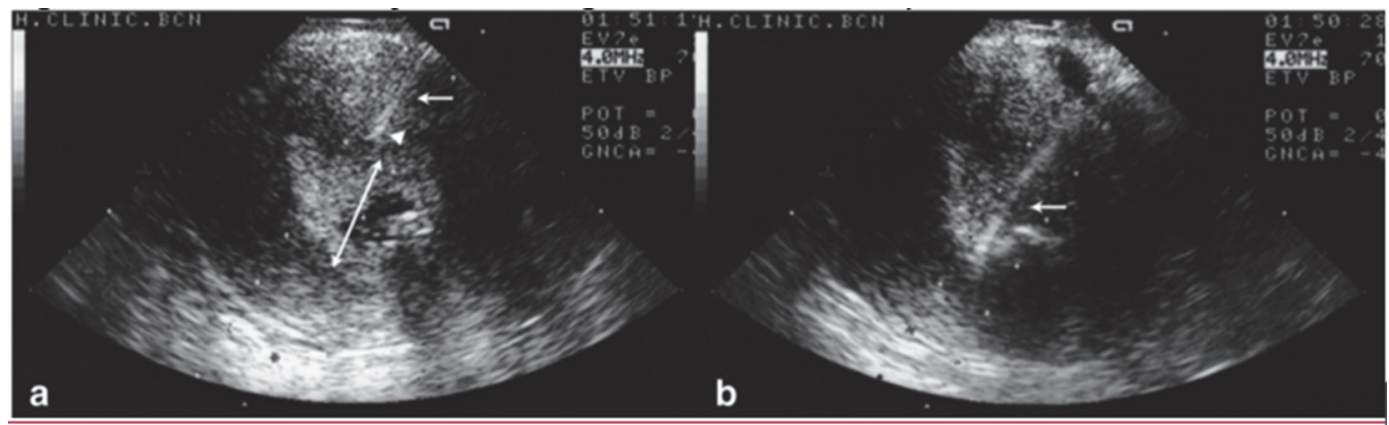

a) Injection into the anterior myometrial wall. b) Injection into the posterior myometrial wall. Arrow: needle; Triangle: Radiotracer cluster.

Abstract 443 Figure 1 Radiotracer injection using the TUMIR technique

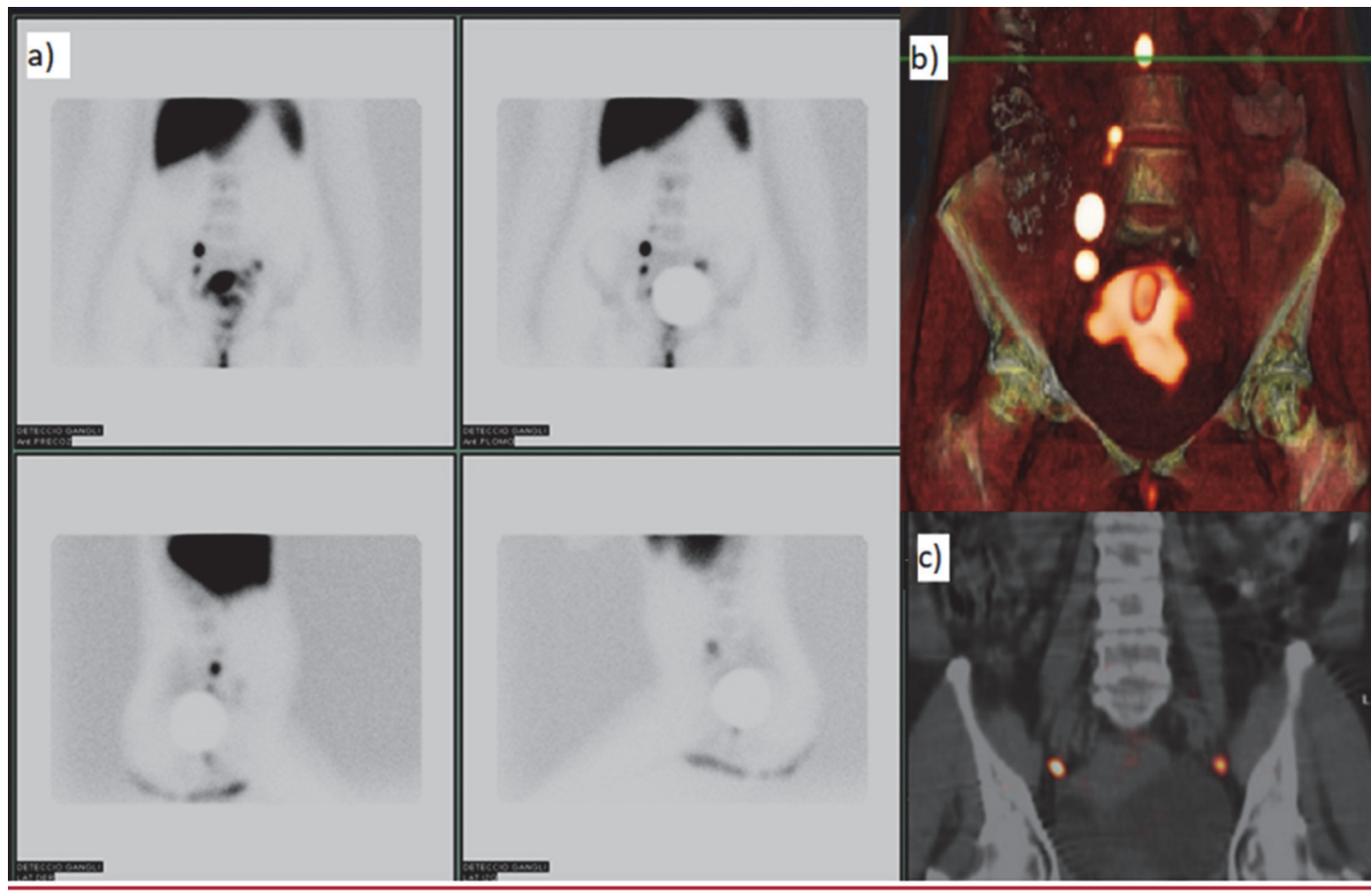

Planar lymphoscintigraphy at $2 \mathrm{~h}$ post injection of the radiotracer (a). Volumetric reconstruction of right hemipelvis SPECT / CT data showing pelvic and paraaortic drainage (b) Coronal section of fused SPECTCT image demonstrating bilateral pelvic drainage (c)

Abstract 443 Figure 2 Planar lymphoscintigraphy and presurgical SPECT/CT

of the patients had positive SLN (1.5\% exclusively paraaortic). The percentage of false negative (FN) was $12.5 \%$.

The intraoperative SLN detection in the RT-ICG group was $68 \%(56 \%$ of pelvic drainage, $33 \%$ of paraortic drainage, without cases with exclusively paraortic drainage). A bilateral drainage was found in $56 \%$ of the cases. The $11.1 \%$ of the patients had a positive SLN without FN cases.

Conclusion Detection of the SLN with RT is slightly higher than with hybrid tracer. The hybrid tracer obtains a higher percentage of SLN with bilateral pelvic drainage than RT and reduces the number of contralateral pelvic lymphadenectomies. The TUMIR technique allows detection of para-aortic SNs in more than $30 \%$ of patients, much higher than that obtained with other techniques.

Disclosures No disclosures.

\section{SHOULD WE RE-STAGE AFTER POSITIVE SENTINEL NODE BIOPSY ON ENDOMETRIAL CANCER?}

${ }^{1}$ Mikel Gorostidi, ${ }^{2}$ Rubén Ruiz Sautua, ${ }^{2}$ Ibon Jaunarena, ${ }^{2}$ Paloma Cobas, ${ }^{2}$ Arantxa Lekuona. ${ }^{1}$ Osakidetza, Hospital Universitario Donostia; Gyn Onc; ${ }^{2}$ Osakidetza, Hospital Universitario Donostia

\subsection{6/ijgc-2020-ESGO.75}

Introduction/Background Approximately $10 \%$ of patients with intraoperative diagnosis of low risk Endometrial Cancer (EC) will suffer an upstage after the definitive histological evaluation of the piece of hysterectomy and bilateral adnexectomy, We aim to explore the results associated with the performance of pelvic and para-aortic lymphadenectomy as restaging these patients that will require a second surgery, and to compare 